\title{
SYMPOSIUM
}

\section{General Symposium}

W.R. BOOT (Convenors). Insights into the measurement of mobile device proficiency across cultures. Gerontechnology 2018;17(Suppl):24s; https://doi.org/10.4017/gt.2018.17.s.024.00 Participants A. PETROVČlČ (SLOVENIA), W.R. BOOT (USA), N.A. ROQUE (USA). Issue Technology proficiency is no longer optional in order to fully participate in society today. In addition to computer proficiency, mobile device (e.g., smartphone, tablet computer) proficiency has become increasingly important to the performance of everyday tasks such as navigation, transportation, communication, shopping, and even healthcare. Unfortunately, there still persists a digital divide in many countries meaning that older adults demonstrate significantly less mobile device ownership and proficiency compared to their younger counterparts, putting them at a disadvantage with respect to using devices and apps that might benefit them. Given the importance of technology proficiency, and especially mobile device proficiency, it has become increasingly important to develop tools to assess older adults' technology proficiency for research and training purposes. Content This symposium will present information on the development and translation of the Mobile Device Proficiency Questionnaire, and the validation of this scale in U.S., Spanish, and Slovenian older adults samples. Structure Roque will begin by describing how and why the MDPQ was developed to better understand mobile device proficiency with respect to the ability of older adults to use mobile devices to support communication and independence. This scale was validated in a sample of 109 U.S. participants and was found to demonstrate high reliability and convergent and divergent validity. Next, Boot will describe the Spanish translation process and validation of the Spanish version of the MDPQ in a sample of 374 participants from Valencia from the ages of 19-93 years of age. Finally, Petrovčič will describe the translation process of the MDPQ into Slovenian, and the collection of data from 50 Slovenian older adults. Crucially, for the first time Petrovčič and colleagues compare selfreported proficiency to objective measures of proficiency in a series of smartphone tasks. Cross-cultural comparisons will describe similarities, and also differences, with respect to the psychometric properties of the scale. Conclusion The symposium will conclude with a brief demonstration of how to use the MDPQ and its different variants for research and training purposes, as well as a discussion of future directions for translation and validation, and projects with the aim of better classifying and characterizing the technology proficiency of older adults across cultures.

Keywords: technology, proficiency, mobile devices, smartphones, tablets, digital divide Address: Florida State University, USA;

E: boot@psy.fsu.edu 


\section{SYMPOSIUM}

\section{General Symposium}

N.A. ROQUE, W.R. BOOT. The development and validation of the Mobile Device Proficiency Questionnaire in U.S. samples. Gerontechnology 2018;17(Suppl):25s; https://doi.org/10.4017/gt.2018.17.s.025.00 Purpose The Mobile Device Proficiency Questionnaire (MDPQ) was developed to help gauge technology proficiency for research and training purposes ${ }^{1}$. Specifically, it was an adaptation of the Computer Proficiency Questionnaire (CPQ) developed by the Center for Research and Education on Aging and Technology Enhancement ${ }^{2}$. This update of the CPQ was necessary to account for recent trends in how people communicate and access the Internet and other services. Both scales (and their short-forms) were created to be quickly and easily completed, even by older adults with very minimal technology experience. Method Originally, data from 109 older adults (ages 65+) were collected, along with data from 40 younger adults (ages 18-29). We will present new data collected since this initial validation sample that further confirm the reliability and validity of the MDPQ, and new analyses that explore the best predictors of mobile device proficiency. Results \& Discussion The initial validation sample, as well as U.S. samples collected afterward, supports the reliability and divergent and convergent validity of the MDPQ. MDPQ score was associated with mobile device experience, and less so with computer experience and other technology experience (though still significantly related). Expected relationships with age were also observed (even among the 65+ group). In general, the MDPQ, its subscales, and short-form, demonstrated excellent reliability (Cronbach's alpha $>.90$ ). This talk will end with a brief tutorial on how to use the MDPQ and how to calculate and interpret MDPQ scores.

\section{References}

1. Roque NA, Boot WR. A new tool for assessing mobile device proficiency in older adults: The mobile device proficiency questionnaire. Journal of Applied Gerontology. 2018;37:131-156.

https://doi.org/10.1177/0733464816642582

2. Boot WR, Charness N, Czaja SJ, Sharit J, Rogers WA, Fisk AD, Mitzner T, Lee C, Nair S. The Computer Proficiency Questionnaire (CPQ): Assessing low and high computer proficient seniors. The Gerontologist. 2015;55:404-411

Keywords: technology, proficiency, mobile devices, smartphones, tablets, digital divide Address: Florida State University, USA;

E: roque@psy.fsu.edu 


\section{SYMPOSIUM}

\section{General Symposium}

A. PETROVČIČ, W.R. BOOT, T. BURNIK, V. DOLNIČAR. Validation of the Mobile Device Proficiency Questionnaire with usability testing in a Slovenian sample. Gerontechnology 2018;17(Suppl):26s; https://doi.org/10.4017/gt.2018.17.s.026.00 Purpose While the age-related gap in the uptake of mobile devices (e.g., tablet computers, smartphones) has been closing, the adoption of mobile applications amongst older adults has remained low and many older adults would benefit from an increase in mobile device proficiency ${ }^{1,2}$. However, to foster older adults' mobile device skills, one first needs to assess their proficiency in a valid manner. Recently, the Mobile Device Proficiency Questionnaire (MDPQ) has been proposed to measure the various dimensions of skills associated with the use of mobile devices ${ }^{3}$. Whilst the MDPQ has been already administrated to older adults, showing excellent construct reliability and validity ${ }^{3}$, no study has yet validated the MDPQ against behavioural task performance criteria. Thus, this study aimed to establish the construct reliability of the MDPQ and determine its criterion validity based on task performance metrics collected in usability tests. Method The research was carried out in 2017 as a confirmatory study aimed, among other objectives, to elucidate the construct reliability and criterion validity of the MDPQ. The full and short version of the MDPQ were translated into Slovenian and administered to a non-probability sample of 50 smartphone users aged 65+, who were tested on an Android and an age-friendly version of a smartphone launcher. The validation of the MDPQ was assessed following a two-stage approach ${ }^{4}$ where covariance-based SEM was used to assess the internal reliability of MDPQ items, while the criterion validity was examined with linear regression models, using MDPQ score as a predictor of task success rate and task efficiency in usability testing. Results \& Discussion Neither of the two MDPQ versions was affected by severe problems of multicollinearity and item redundancy. However, the construct reliability was smaller than expected, as many of the parameter estimates of the items in the measurement model were not significant. In terms of criterion validity, the linear regression models with long and short versions of the MDPQ as predictors of task performance metrics yielded high values of explained variance, indicating that the MDPQ could successfully predict the total success and efficiency of older adults while operating the two versions of a smartphone launcher. Interestingly, the criterion validity of the short version of MDPQ was not significantly lower when compared with the long version, suggesting that the former might be desired when respondent burden is a major concern in a survey.

\section{References}

1. Berenguer A, Goncalves J, Hosio S, Ferreira D, Anagnostopoulos T, Kostakos V. Are smartphones ubiquitous?: An in-depth survey of smartphone adoption by seniors. IEEE Consumer Electronics Magazine. 2017;6(1):104-110

2. Petrovčič A, Slavec A, Dolničar V. The ten shades of silver: Segmentation of older adults in the mobile phone market. International Journal of Human-Computer Interaction. 2017;1-16.

https://doi.org/10.1080/10447318.2017.1399328

3. Roque NA, Boot WR. A new tool for assessing mobile device proficiency in older adults: The mobile device proficiency questionnaire. Journal of Applied Gerontology. 2018. https://doi.org/10.1177/0733464816642582

4. Diamantopoulos A, Siguaw JA. Formative versus reflective indicators in organizational measure development: A comparison and empirical illustration. British Journal of Management. 2016;17:263-282

Keywords: criterion validity, mobile device proficiency questionnaire, older adults, smartphones, usability testing

Address: University of Ljubljana, Slovenia;

E: andraz.petrovcic@fdv.uni-lj.si 


\section{SYMPOSIUM}

\section{General Symposium}

C. MORET-TATAY, W.R. BOOT, M.E. GUTIÉRREZ-MARCO, N. CHARNESS. Translation of the Mobile Device and Computer Proficiency Questionnaires into Spanish, and Spanish validation. Gerontechnology 2018;17(Suppl):27s; https://doi.org/10.4017/gt.2018.17.s.027.00 Purpose As ICT spreads globally, differences among cultures might appear. More precisely, smartphone and computer use seem to vary considerably from one country to another. According to the European Eurobarometer', the largest increases have been observed in Spain, Italy, Croatia and Hungary respectively, while other countries such as the UK, Sweden and Luxembourg might be approaching a plateau. In order to help characterize and explain different adoption rates and their causes, we believe more research is needed that examines mobile and computer proficiency across countries. To help achieve this goal, the Mobile Device Proficiency Questionnaire (MDPQ) ${ }^{2}$ and the Computer Proficiency Questionnaire $(\mathrm{CPQ})^{3}$ for older adults were translated into Spanish, including the full and brief forms of each measure. Method This research was carried out in 2016 as an exploratory study aimed to adapt the CPQ and MDPQ tools for an older Spanish population. Both full and short versions of MDPQ were translated into Spanish and then back-translated into English for review and modification. Then, a convenience sample of 374 participants ranging in age from 19-93 years of age was selected to complete the questionnaires. Results \& Discussion Consistent with results using the English versions of the questionnaires, the scales and their subscales were found to be highly reliable and valid measures of mobile device and computer proficiency in Spanish older adults. However, in contrast to earlier studies, the factor structure for both questionnaires simplified into two factors for the Spanish sample. We conclude that both Spanish versions of the MDPQ and CPQ can be employed as useful tools for measuring mobile device and computer proficiency in the Spanish older adult population for research and training purposes. Moreover, these newly translated measures will assist in understanding differences in technology proficiency among older adults in different countries.

\section{References}

1. European Commission. (2015). Eurobarometer 423, October February 2015. TNS OPINION \& SOCIAL, Brussels [Producer]; GESIS Data Archive: EB82.2

2. Roque NA, Boot WR. A new tool for assessing mobile device proficiency in older adults: The Mobile Device Proficiency Questionnaire. Journal of Applied Gerontology. 2018;37:131-156; https://doi.org/10.1177/0733464816642582

3. Boot WR, Charness N, Czaja SJ, Sharit J, Rogers WA, Fisk AD, Mitzner T, Lee CC, Nair S. Computer Proficiency Questionnaire: Assessing low and high computer proficient seniors. The Gerontologist. 2015;55:404-411; https://doi.org/10.1093/geront/gnt117

Keywords: technology, proficiency, mobile devices, smartphones, tablets, digital divide Address: Florida State University, USA;

E: boot@psy.fsu.edu 